\title{
Reflection of VLF Radio Waves From an Inhomogeneous Ionosphere. Part I. Exponentially Varying Isotropic Model ${ }^{1}$
}

\author{
James R. Wait and Lillie C. Walters
}

Contribution from Central Radio Propagation Laboratory, National Bureau of Standards, Boulder, Colo.

(Received January 3, 1963)

\begin{abstract}
The oblique reflection of VLF radio waves from a continuously stratified ionized medium is considered. The profile of the effective conductivity is taken to be of an exponential form. This is a fair representation for the actual $D$ layer of the ionosphere. It is shown that the gradient of the conductivity change has a marked effect on the reflection characteristies.
\end{abstract}

\section{Introduction}

In the study of VLF radio wave propagation it is often assumed that the ionosphere can be regarded as a sharply bounded medium on its underside. This step may be considered reasonable in view of the long wavelength and the relatively rapid change of the electron density of the $D$ region. Furthermore, experimental data are often in accord with calculations based on this model. The general agreement is particularly good at highly oblique incidence provided that appropriate corrections are made for earth curvature. Nevertheless, there are many occasions when the sharply bounded model appears to be inadequate. Therefore, it is worthwhile to consider a more realistic model of the lower ionosphere.

On examining much of the recent literature on the characteristics of the lower $D$ layer, it appears that the effective dielectr. constant of the medium can be well approximated by an exponential function. Then, to within this approximation, the relative permittivity may be written in the form

$$
K(z)=K_{0}\left(1-i \frac{1}{L} \exp \beta z\right)
$$

where $K_{0}$ is a reference permittivity, and $L$ and $\beta$ are constants. The level $z=0$ may be defined as the reference level and thus

$$
K(0)=K_{0}\left(1-i \frac{1}{L}\right)
$$

which is a familiar form.

1 The work in this paper was supported by the Advanced Research Projects Agency, Washington 25, D.C., under ARPA Order No. 183-62.
Under the assumptions that the angular frequency $\omega$ is much less than both the collision frequency $\nu$ and the plasma frequency $\omega_{0}$, it is known that [Wait, 1962]

$$
K_{0}=1 \text { and } L=\frac{\omega \nu}{\omega_{0}^{2}}
$$

provided that the earth's magnetic field can be neglected. The latter assumption is strictly valid only if $\nu$ is somewhat greater than the gyrofrequency $\omega_{H}$. In actual cases, however, the isotropic assumption is useful even when $\nu$ is of the order of $\omega_{H}$, provided the magnetic field is not transverse to the direction of propagation [Crombie, 1961; Johler and Harper, 1962]. For the purposes of the present paper, the influence of the terrestrial magnetic field is not considered.

The constant $\beta$ in the exponent of the equation for $K(z)$ is a measure of the sharpness of the gradient. For example, when $\beta=1 \mathrm{~km}^{-1}$, it means that the ratio $\omega_{0}^{2} / \nu$ or $N / \nu$ increases by a factor 2.71 for each $\mathrm{km}$ of vertical height. The best available information on expected values of $\beta$ can be found in the recent work on pulse cross modulation [Barrington et al., 1962] and from recent rocket measurements [Kane, 1962]. From these results it appears that $\beta$ is of the order of $1 / 2 \mathrm{~km}^{-1}$ for quiet daytime conditions although it may differ by a factor of two or more at certain times. In the present study it appears to be desirable to allow $\beta$ to vary from 0.3 to 3.0 to encompass most cases of interest.

\section{Background Theory}

The propagation of electromagnetic waves in a medium whose permittivity $\epsilon(z)$ varies in an exponential manner has been studied extensively. The usual case considered is for horizontal polarization where the electric vector is parallel to the stratifica- 
tion. For example, if the electric vector has only a $y$ component $E_{y}$, it is a simple matter to show that [Wait, 1962]

$$
\left[\frac{d^{2}}{d z^{2}}+k^{2}\left(K(z)-S^{2}\right)\right] E_{y}=0,
$$

when the field varies in the $x$ direction according to $\exp (-i k S x)$, where $S$ is a dimensionless constant. Here $k=\sqrt{\epsilon_{0} \mu_{0}} \omega$ is the free-space wave number and $\epsilon_{0}$ and $\mu_{0}$ are the permittivity and permeability of free space, respectively.

At a sufficiently large negative value of $z$, the relative permittivity $K(z)$ becomes unity, and thus, $E_{y}$ satisfies

$$
\left(\frac{d^{2}}{d z^{2}}+k^{2} C^{2}\right) E_{y}=0
$$

where $C^{2}=1-S^{2}$. The general solution of this equation has the form

$$
E_{y}=E_{0}\left(e^{-i k C z}+R_{h} e^{+i k C z}\right) e^{-i k S x},
$$

when $E_{0}$ and $R_{h}$ are constants. Recognizing that the solution of (4) must reduce to (5), for $z$ tending to $-\infty$, it may be shown that [Wait, 1962]

$$
R_{h}=-\left(\frac{k}{\beta}\right)^{2_{\nu}} \frac{1}{(-i L)^{\nu_{0}}} \frac{\left(-\nu_{0}\right) !}{\left(\nu_{0}\right) !}
$$

where $\nu_{0}=2 i k C / \beta$.

The term $E_{0} e^{-i k C z} e^{-i k S x}$ may be regarded as an incident wave whose direction of propagation makes angle $\theta$ with the $z$ axis where $C=\cos \theta$ or $S=\sin \theta$. Then $E_{0} R e^{+i k C z} e^{-i k S x}$ can be interpreted as a reflected wave and $R$ can be defined as the reflection coefficient of the exponential layer. It should be stressed that this reflection coefficient, while referred to the level $z=0$, is only valid in the free space region at large negative values of $z$.

The reflection coefficient $R_{h}$, given by (7), may be conveniently written in the form

$$
R_{h}=\exp \left(-\frac{2 \pi^{2}}{\lambda_{0} \beta} C\right) \exp i \Phi,
$$

where

$$
\Phi=\frac{8 \pi}{\lambda_{0} \beta} C \log \left(\frac{2 \pi}{\lambda_{0} \beta L^{1 / 2}}\right) \pm \pi+2 \arg \left[\left(-i \frac{4 \pi C}{\lambda_{0} \beta}\right) !\right]
$$

where $\lambda_{0}=2 \pi / k$ is the free-space wavelength. Thus, the amplitude of the reflection coefficient is given by the very simple form

$$
\left|R_{h}\right|=\exp \left(-\frac{2 \pi^{2}}{\lambda_{0} \beta} C\right),
$$

which is independent of $L$. On the other hand, the phase factor $\Phi$ is relatively complicated since it involves the factorial function of imaginary argument. Nevertheless, (8), for the complex value of $R_{h}$ can be used to obtain quantitative results for exponentialtype layers.

\section{Extension to Vertical Polarization}

In the VLF radio problem one is mainly interested in vertical polarization such that the magnetic vector is parallel to the stratification. For example, if the magnetic field has only a $y$ component, $H_{y}$, and assuming again that the fields vary in the $x$ direction according to $\exp (-i k S x)$, it follows that [Wait, 1962]

$$
\left[\frac{d^{2}}{d z^{2}}-\frac{1}{K(z)} \frac{d K(z)}{d z} \frac{d}{d z}+k^{2}\left(K-S^{2}\right)\right] H_{y}=0 .
$$

Intrinsically, this is a more complicated form than (4) for horizontal polarization.

In the region of large negative $z$ the (10) reduces to the elementary form

$$
\left(\frac{d^{2}}{d z^{2}}+k^{2} C\right) H_{y}=0,
$$

and thus

$$
H_{y}=H_{0}\left(e^{-i k C z}+R_{v} e^{+i k C z}\right) e^{-i k S x},
$$

where $H_{0}$ is a constant and $R_{v}$ is, by definition, the reflection coefficient for vertical polarization.

Unfortunately, for the exponentially varying permittivity of the form defined by (1), it does not appear to be possible to obtain a closed form expression for $R_{v}$. For this reason, the required quantitative results were obtained by a numerical method. Essentially, the procedure is to replace the continuous $K(z)$ profile by a finite number of steps. The situation is illustrated in figure 1 where the function

$$
\frac{1}{L(z)}=\frac{1}{L} \exp \beta z
$$

is shown plotted versus $z$ along with its step approximation. Thus, between the limits $z=-z_{0}$ and $z=+T$, the medium is divided into $M$ homogeneous layers of width $h_{1}, h_{2} \ldots h_{m}, \ldots h_{M-1}, h_{M}$. The value of $L(z)$ in each of these slabs is then replaced by a

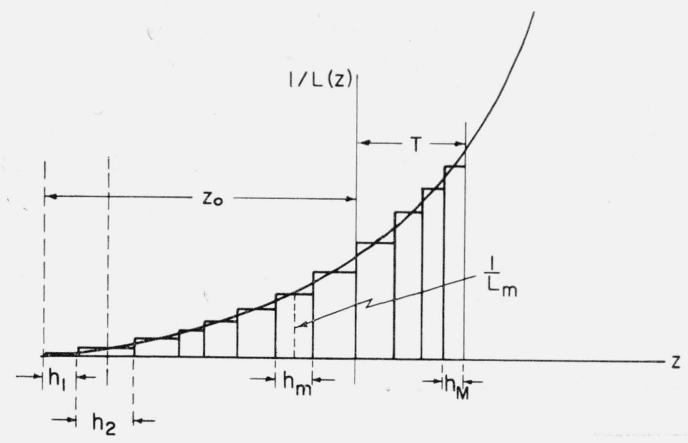

Figure 1. The step approximation to an exponential profile. 
constant value $L_{m}$. The method is really equivalent to the usual method [Budden, 1961] of numerically integrating the basic differential equations.

The problem now boils down to finding the wave impedance $Z_{1}$ at $z=-z_{0}$ in terms of the properties of all the individual slabs. Here $Z_{1}=\frac{E_{x}}{H_{y}}$ which is to be evaluated at $z=-z_{0}$. The reflection coefficient $R_{v}$, which is referred to the level $z=0$, is then found from

$$
R_{v}=\frac{C-Z_{1} / \eta_{0}}{C+Z_{1} / \eta_{0}} \exp \left[-i \frac{4 \pi}{\lambda_{0}} C z\right],
$$

for $z=-z_{0}$, and where $\eta_{0}=\sqrt{\mu_{0} / \epsilon_{0}}=120 \pi$. Here $z_{0}$ is chosen sufficiently large that $1 / L(z)$ may be regarded as zero. (Strictly speaking, $z_{0} \rightarrow \infty$.)

\section{Iterative Process}

From the theory of wave propagation in stratified media [e.g., Wait, 1962], it is known that $Z_{1}$ can be obtained by a series of iterative processes. Thus

where

$$
Z_{1}=K_{1} Q_{1}
$$

$$
\begin{gathered}
Q_{1}=\frac{K_{2,1} Q_{2}+\tanh D_{1}}{1+K_{2,1} Q_{2} \tanh D_{1}}, \\
Q_{2}=\frac{K_{3,2} Q_{3}+\tanh D_{2}}{1+K_{3,2} Q_{3} \tanh D_{2}} \\
\ldots \\
Q_{m}=\frac{K_{m+1, m} Q_{m+1}+\tanh D_{m}}{1+K_{m+1, m} Q_{m+1} \tanh D_{m}},
\end{gathered}
$$

and so on. The various factors are defined below. The parameter $D_{m}$ which is a measure of the thickness of an individual slab is defined by

$$
D_{m}=\frac{i 2 \pi h_{m}}{\lambda_{0}} N_{m}\left(1-\frac{S^{2}}{N_{m}^{2}}\right)^{1 / 2},
$$

where $N_{m}$ is the refractive index of the $m$ th slab given by ${ }^{2}$

$$
N_{m}=\left(1-\frac{i}{L_{m}}\right)^{1 / 2}
$$

The quantity $K_{m+1, m}$ is the ratio of the wave impedances of layer $m+1$ and layer $m$. Thus

$$
K_{m+1, m}=\frac{K_{m+1}}{K_{m}}
$$

where

$$
K_{m}=\frac{\eta_{0}}{N_{m}}\left(1-\frac{S^{2}}{N_{m}^{2}}\right)^{1 / 2},
$$

\footnotetext{
2 The square roots are defined such that $D_{m}=\left(\frac{i 2 \pi h_{m}}{\lambda_{0}}\right) N_{m}$ if $N_{m} \rightarrow \infty$, while $D_{m}=\left(\frac{i 2 \pi h_{m}}{\lambda_{0}}\right) C$ if $N_{m} \rightarrow 1$. Furthermore, $N_{m} \simeq\left(1 / L_{m}\right)^{1 / 2} e^{-i \pi / 4}$ if $\left|N_{m}\right|>>1$ and $N_{m}=1$ if $L_{m} \rightarrow \infty$.
}

is the wave impedance in the $m$ th layer. Alternately,

$$
K_{m+1, m}=\frac{\left(1-\frac{i}{L_{m}}\right)}{\left(1-\frac{i}{L_{m+1}}\right)}\left[\frac{C^{2}-\frac{i}{L_{m+1}}}{C^{2}-\frac{i}{L_{m}}}\right]^{1 / 2} .
$$

Finally, because the quantities $Q_{m}$ are given in terms of $Q_{m+1}$ it is necessary to know the initial value $Q_{M+1}$ at $z=T$. For example,

$$
Q_{M}=\frac{K_{M+1, M} Q_{M+1}+\tanh D_{M}}{1+K_{M+1, M}\left[\tanh D_{M}\right] Q_{M+1}}
$$

and similarly, $Q_{M-1}$ is expressed in terms of $Q_{M}$. The process is continued until $Q_{1}$ is obtained.

In some problems of this kind it is not necessary to know precisely the value of $Q_{M+1}$. However, the economy of the calculations is greatly improved if a good starting value of $Q_{M+1}$ is known. Recognizing that the wave impedance at $z=T$ is given by

$$
Z_{M+1}=\frac{K_{M+1}}{\eta_{0}} Q_{M+1}
$$

and utilizing the condition $\left|N_{M+1}\right|>>1$, it follows from previous work [Wait, 1962] that

$$
Q_{M+1} \simeq \frac{K_{0}\left[\sqrt{i} \frac{2 \pi}{\lambda_{0}} N_{M+1} \frac{2}{\beta}\right]}{K_{1}\left[\sqrt{i} \frac{2 \pi}{\lambda_{0}} N_{M+1} \frac{2}{\beta}\right]},
$$

where $K_{0}$ and $K_{1}$ are modified Bessel functions.

In a practical sequence of calculations it is necessary to choose $T$ large enough that the final result for $R_{v}$ is insensitive to further changes in $T$. For example, if $\beta=1 \mathrm{~km}^{-1}$ and $\lambda_{0}=15 \mathrm{~km}$, it was found that $T=4 \mathrm{~km}$ was sufficiently large to obtain four-figure accuracy in $R_{v}$. Furthermore, for some values of $\beta$ and $\lambda_{0}$, it was found that $R_{v}$ approaches a constant as $z_{0}$ was increased to $20 \mathrm{~km}$. In general, for smaller values of $\beta$ it was necessary to increase $T$ and $z_{0}$ to larger values in order to achieve stability of the final results. The width of the steps, $h_{m}$, must also be chosen sufficiently small to achieve an adequate simulation of the smooth profile. Generally, it was found that $\left|D_{m}\right|<10^{-2}$ was a satisfactory criterion.

\section{Discussion of Reflection Coefficient Calculations}

Using the basic definition of the reflection coefficient $R_{v}$, given by (14), the amplitude and phase of $R_{v}$ were calculated for a wide range of conditions. While, strictly speaking, $R_{v}$ is defined as the limit for $z=-z_{0}$, where $z_{0} \rightarrow \infty$, it is of interest to first demonstrate how $R_{0}$ approaches this limit. An example of such calculations is shown in figures $2 \mathrm{a}$ and 2b. Here $C=0.1$, corresponding to highly oblique incidence, and $\lambda_{0}=15 \mathrm{~km}$, or $f=20 \mathrm{kc} / \mathrm{s}$. 

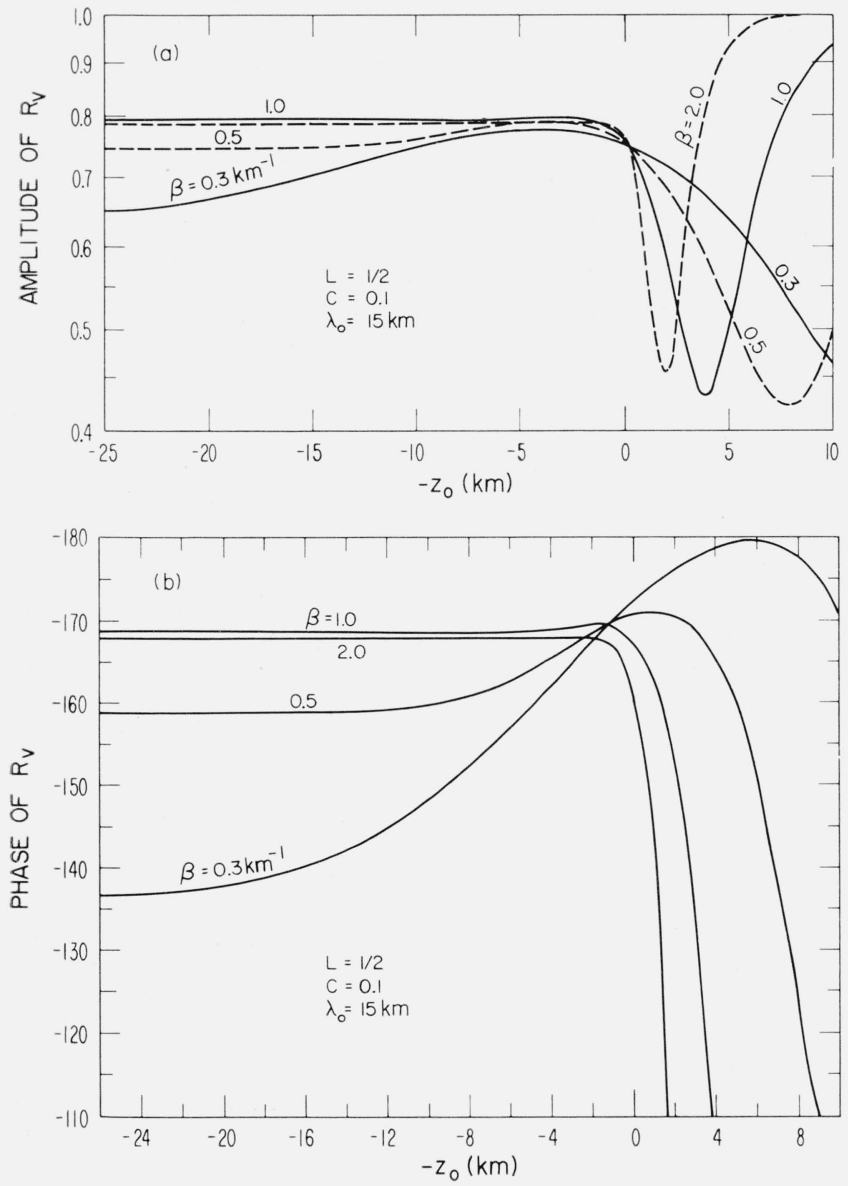

Figure 2. The reflection coefficient as a function of height for an exponential profile and a fixed angle of incidence.

Various values of $\beta$ are shown on the curves. It is immediately apparent that for sufficiently negative values of $z$ (i.e., sufficiently far below the layer), both the amplitude and phase of $R_{v}$ approach a limit.

Actually, the curves in figures $2 \mathrm{a}$ and $2 \mathrm{~b}$ have more than just a mathematical interest. Physically, the $R_{v}$, corresponding to $z=-z_{0}$ where $z_{0}$ is finite, is the reflection coefficient for a permittivity profile of the form

$$
\begin{aligned}
K(z) & =K_{0}\left[1-\frac{i}{L} e^{\beta z}\right] \text { for } z>-z_{0} \\
& =0 \text { for } z<-z_{0} .
\end{aligned}
$$

Therefore, these curves describe reflection from a sharply bounded ionosphere which behaves exponentially above its lower edge. Of course, as $z_{0}$ tends to $\infty$, the discontinuous profile becomes a continuous exponential. It is rather interesting to observe that as the discontinuity is moved from above the reference level $(z=0)$ to below, the reflection coefficient passes through a minimum. This is related to a Brewster angle phenomenon

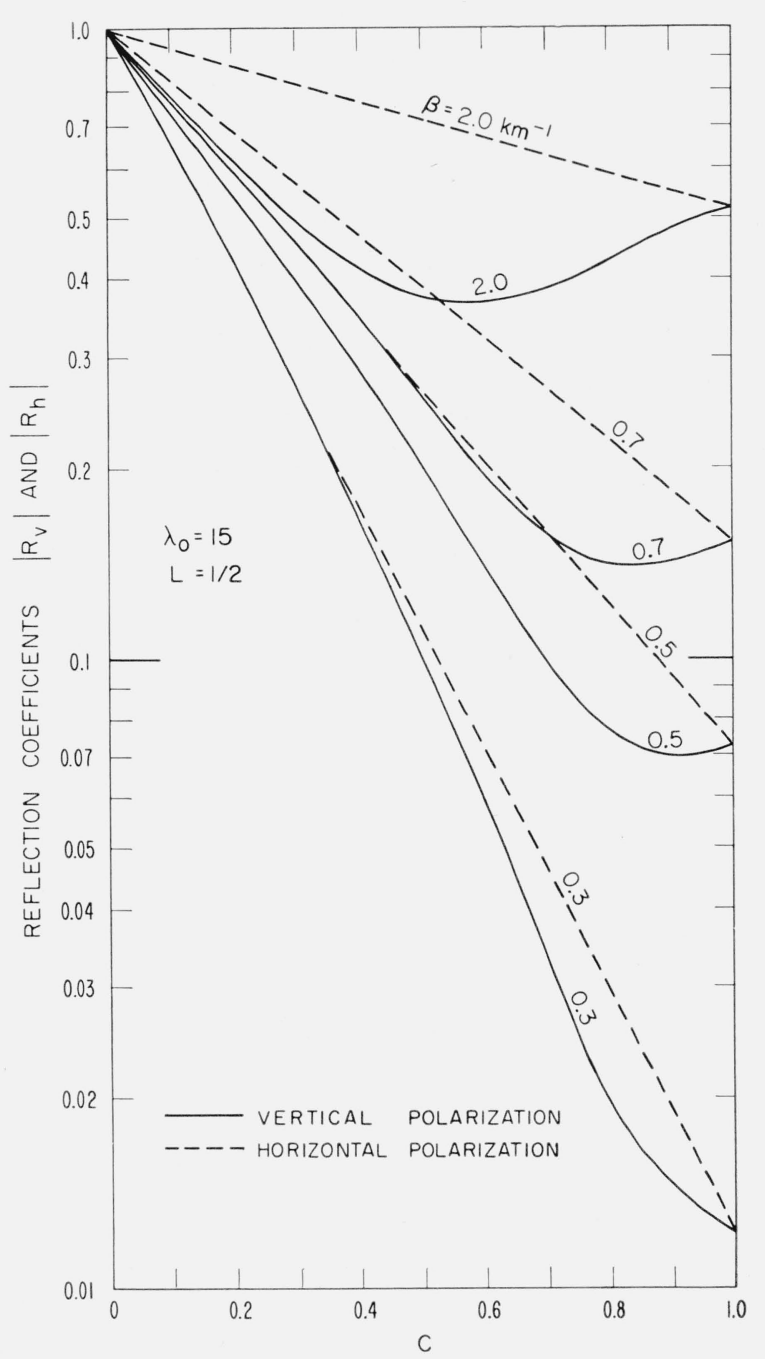

Figure 3. The amplitude of the reflection coefficients for vertical and horizontal polarization as a function of $\mathrm{C}$ which is the cosine of the angle of incidence.

since to the right of this minimum in figure 2 a the reflection is metalliclike while, to the left, the reflection is dielectriclike.

For the remainder of the present paper, attention will be confined to the limiting value of $R_{v}$ far below the reference level $z=0$ (i.e., $z \cong-z_{0} \cong-\infty$ ). On the other hand, it should be emphasized that $R_{v}$ is always referred to the level $z=0$.

The magnitude of the reflection coefficients $R_{v}$ and $R_{h}$ are shown plotted in figure 3 for vertical and horizontal polarization, respectively. The values of $\left|R_{v}\right|$ were obtained from (14) using the multislab model described. The values of $\left|R_{h}\right|$ were calculated directly from (9) and, on this log-linear scale they are merely straight lines. As an important check, the value of $\left|R_{h}\right|$ was also obtained from a multislab model using the same procedure as described for vertical polarization. Within four-figure accuracy, the values of $\left|R_{h}\right|$ obtained by the two methods were the same. 
A number of significant features are evident in figure 3 . In the first place, $\left|R_{v}\right|$ exhibits a Brewster angle phenomenon, provided $\beta$ is sufficiently large. Here the reflection process is dielectriclike at grazing angles and is more or less metalliclike at normal incidence. Of course, $\left|R_{h}\right|$ does not exhibit this phenomenon. However, for very small values of $\beta$, corresponding to a relatively slowly varying medium, the curves for $\left|R_{h}\right|$ and $\left|R_{v}\right|$ become rather close to one another. This is consistent with the optical behavior of waves in an inhomogeneous medium. In fact, by a direct application of the phase integral method of Eckerskey, the equation for $\left|R_{h}\right|$ is found also to be applicable to $\left|R_{v}\right|$. The use of the phase integral method for such applications is necessarily restricted to slowly varying media [Wait, 1962]. It is apparent, that for VL.F radio waves, where $\beta$ is of the order of 0.5 , the phase integral method is inapplicable to vertical polarization.

Another interesting feature of figure 3 is the near linear dependence of all the curves for small values of $C$. Fortunatelv, it is just these values which are important in the long-distance propagation of VLF radio waves. The linearity of the phase curves for $\mathbf{R}_{v}$ are indicated in figure 4 for the same conditions. Here, it is evident that they all approach $-180^{\circ}$ at grazing incidence. The phase curves for $\mathbf{R}_{h}$ exhibit a similar property but they are not shown here since they have only an academic interest at VLF.

The variation of the reflection coefficient $\mathbf{R}_{v}$ as a function of the gradient parameter $\beta$ is illustrated in figures $5 \mathrm{a}$ and $5 \mathrm{~b}$ at oblique incidence. It is rather remarkable that $\left|R_{v}\right|$ is relatively insensitive to $\beta$ if it is in the range from 0.7 to 3.0. Furthermore, it appears that $\left|R_{v}\right|$ has a broad maximum for $\beta$ approximately equal to 1.2 .

The general behavior of the amplitude and phase of the reflection coefficients at highly oblique incidence suggests that, if $R_{v}$ is written in the form

$$
R_{\eta}=-\exp (\alpha C)
$$

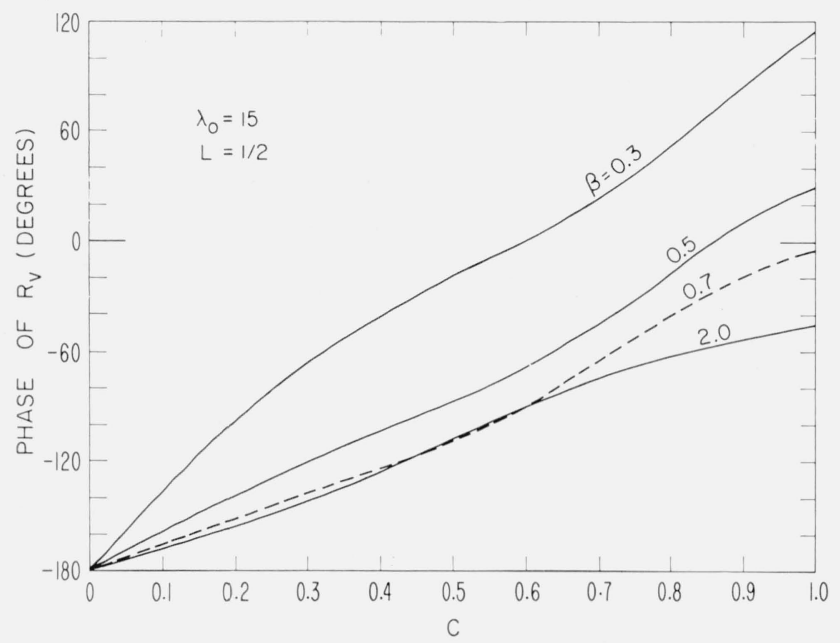

FIGURE 4. The phase of the reflection coefficient for vertical polarization as a function of $\mathrm{C}$. the function $\alpha$ should be almost independent of $C$. Writing

$$
\alpha=\alpha_{1}+i \alpha_{2},
$$

where $\alpha_{1}$ and $\alpha_{2}$ are real, it is a simple matter to compute the complex coefficient $\alpha$ from the numerical data of $R_{v}$. The results are shown in figures $6 \mathrm{a}$ and $6 \mathrm{~b}$ where $\left(-\alpha_{1}\right)$ and $\alpha_{2}$, respectively, are plotted as a function of $C$ in the range 0.05 to 0.30 . It is apparent that over this range of $C$, the coefficient $\alpha$ can be regarded essentially as a complex constant.

For all the results given in the foregoing figures, it has been assumed that $L=1 / 2$ and $\lambda_{0}=15$. Actually, the results for the magnitude of $R_{v}$ and $R_{h}$ do not depend on $L$. In fact, $\left|R_{v}\right|$ and $\left|R_{h}\right|$ are identical for $\beta \lambda_{0}=$ constant. Furthermore, the phase of the reflection coefficients is also simply related although the situation is slightly complicated by the choice of the reference level where $L$ is required to have a special value. In fact, it is convenient to choose the reference level (i.e., $z=0$ ) so that

$$
L=\frac{\omega \nu}{\omega_{0}^{2}}=\frac{1}{2} \times \frac{15}{\lambda_{0}} .
$$

In this way, the results can be readily compared as a function of frequency or wavelength. At the reference level $z=0$, the effective conductivity parameter $\omega_{i}$ or $\omega_{0}^{2} / \nu$ has the value $2.51 \times 10^{5}$.

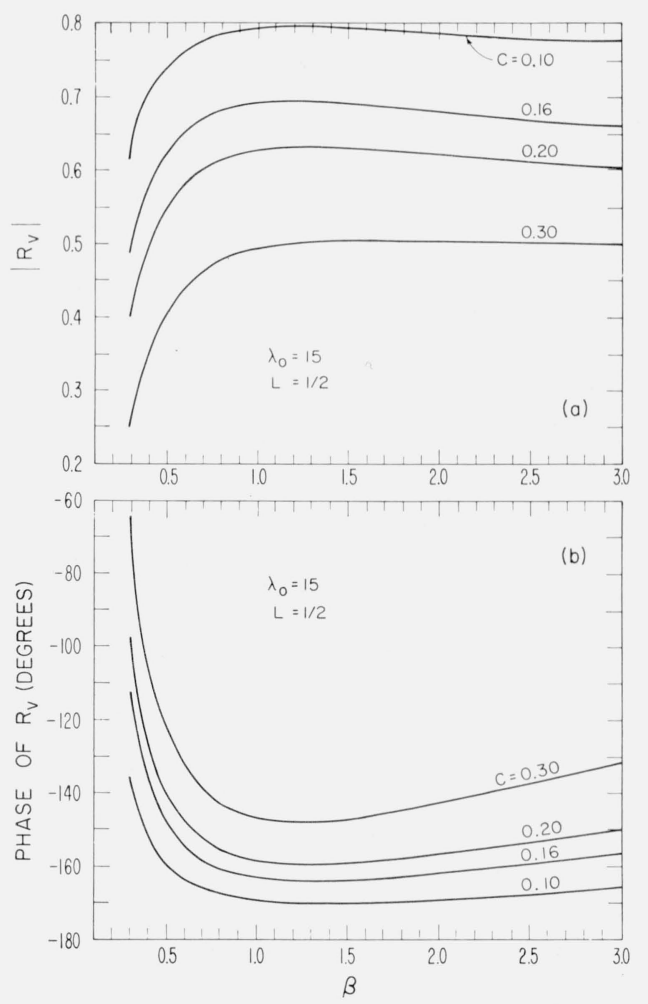

Figure 5. The reflection coefficient as a function of the gradient parameter $\beta$ for an exponential profile. 

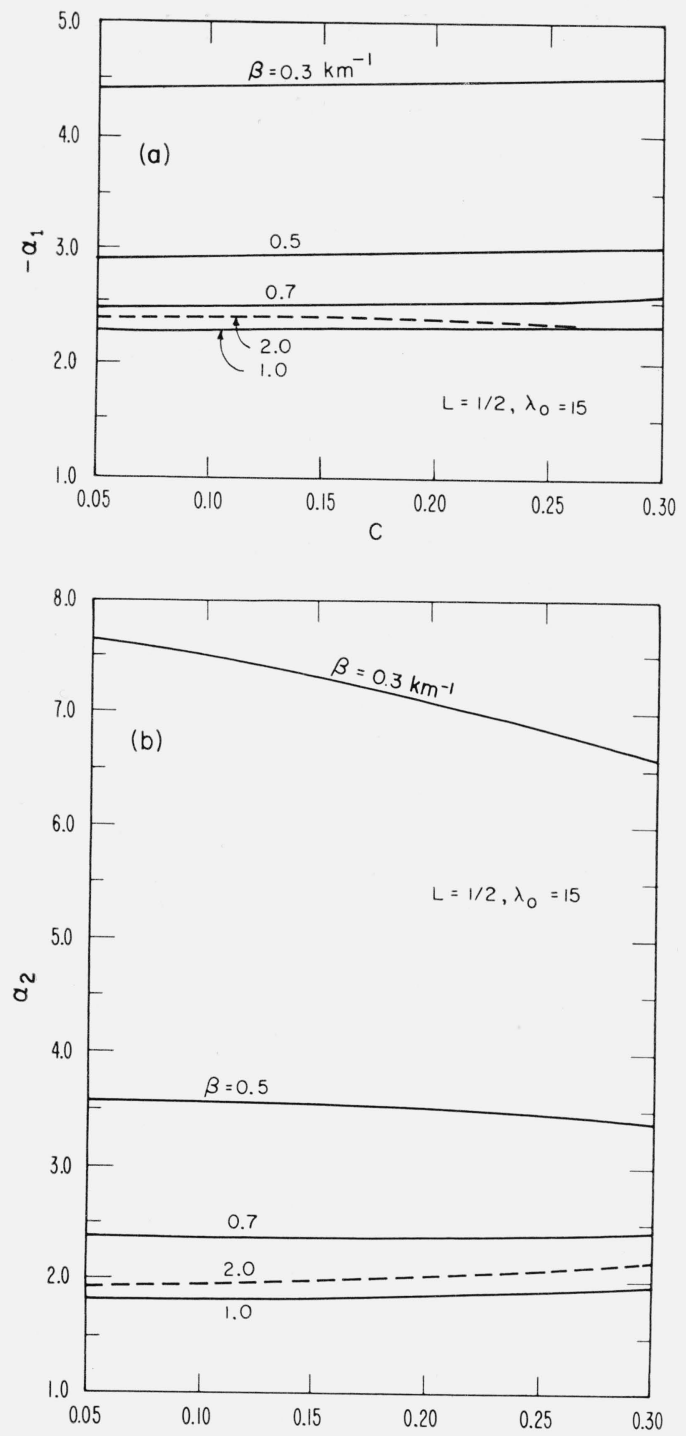

FIgure 6. The real and imaginary parts of the function $\alpha$ defined by $\mathrm{R}_{\mathrm{v}}=-\exp (a \mathrm{C})$ for $\lambda_{\mathrm{o}}=15$ and various values of $\beta$

For wavelengths other than $\lambda_{0}=15$, it is also found that $R_{v}$ may be approximated by the function$\exp (\alpha C)$ where $\alpha$ is approximately a complex constant. To illustrate the wavelength dependence, $-\alpha_{1}$ and $\alpha_{2}$ are plotted as a function of $\lambda_{0}$ in figures $7 \mathrm{a}$ and $7 \mathrm{~b}$, respectively. For these curves, $C$ is chosen to be 0.16 ; the corresponding curves for other values of $C$ in the range 0.10 to 0.20 are almost indistinguishable. The ordinate in figure $7 \mathrm{a}$ is simply related to the magnitude of the reflection coefficient and, thus, small values of $-\alpha_{1}$ are associated with high reflection coefficients. It is apparent that for small values of $\beta$ corresponding to a diffuse layer, the reflection coefficient becomes very small for the shorter wavelength. On the other hand, for a rapidly varying layer, corresponding to large values of $\beta$, the reflection coefficient decreases with increasing wavelength. For intermediate values of $\beta$, the reflection coefficient has a minimum in the wavelength range between 10 and $30 \mathrm{~km}$. For example, when $\beta=1.0$, the optimum wavelength is about 17 $\mathrm{km}$ or approximately $18 \mathrm{kc} / \mathrm{s}$.

The curves for $\alpha_{2}$ in figure $7 \mathrm{~b}$ also have a particular significance. Noting that

$$
\arg R_{v}=-\pi+\alpha_{2} C,
$$

it is apparent that $\alpha_{2} C$ is the phase shift resulting from the imperfect reflecting properties of the exponential layer. To attach a physical meaning to this term it is often desirable to imagine the reflection taking place at a height $\Delta z_{1}$ below the level $z=0$. In this case, $\Delta z_{1}$ is chosen so that the arg of $R_{v}$ is always $-\pi$. Clearly,

$$
\alpha_{2}=2 k \Delta z_{1}=4 \pi \Delta z_{1} / \lambda_{0},
$$

or

$$
\Delta z_{1}=\alpha_{2} \lambda_{0} /(4 \pi)
$$

For example, at $\lambda_{0}=15 \mathrm{~km}$ (i.e., $20 \mathrm{kc} / \mathrm{s}$ ) and $\beta=1$, the effective height of reflection is depressed by approximately $3 \mathrm{~km}$. For smaller values of $\beta$, it is seen from figure $7 \mathrm{~b}$ that $\Delta z_{1}$ may be much greater.

\section{Final Remarks}

The results, for the coefficients $\alpha_{1}$ and $\alpha_{2}$, given here may be introduced into the waveguide mode theory. In this way, attenuation rates and phase velocities of the modes may be obtained. This is a valid subject for another paper.

The influence of nonexponential profiles also appears to be a subject worthy of some attention. Using the iterative method described in this paper, it appears that any reasonably smooth profile may be treated in a straightforward manner. Also, with some modification, the technique may be used to evaluate the effect of discontinuities in gradients and local regions of excess ionization.

General conclusions about the influence of the profile of the lower ionosphere on VLF propagation must await completion of extensive and systematic calculations. It is hoped to report such results in the near future. Detailed comparisons of theory and experiment are also deferred until these more complete computations become available.

We thank Mrs. Carolen Jackson and Mrs. Eileen Brackett for their assistance in the preparation of this paper. In addition, we would like to thank Douglass D. Crombie for his useful suggestions and comments. 

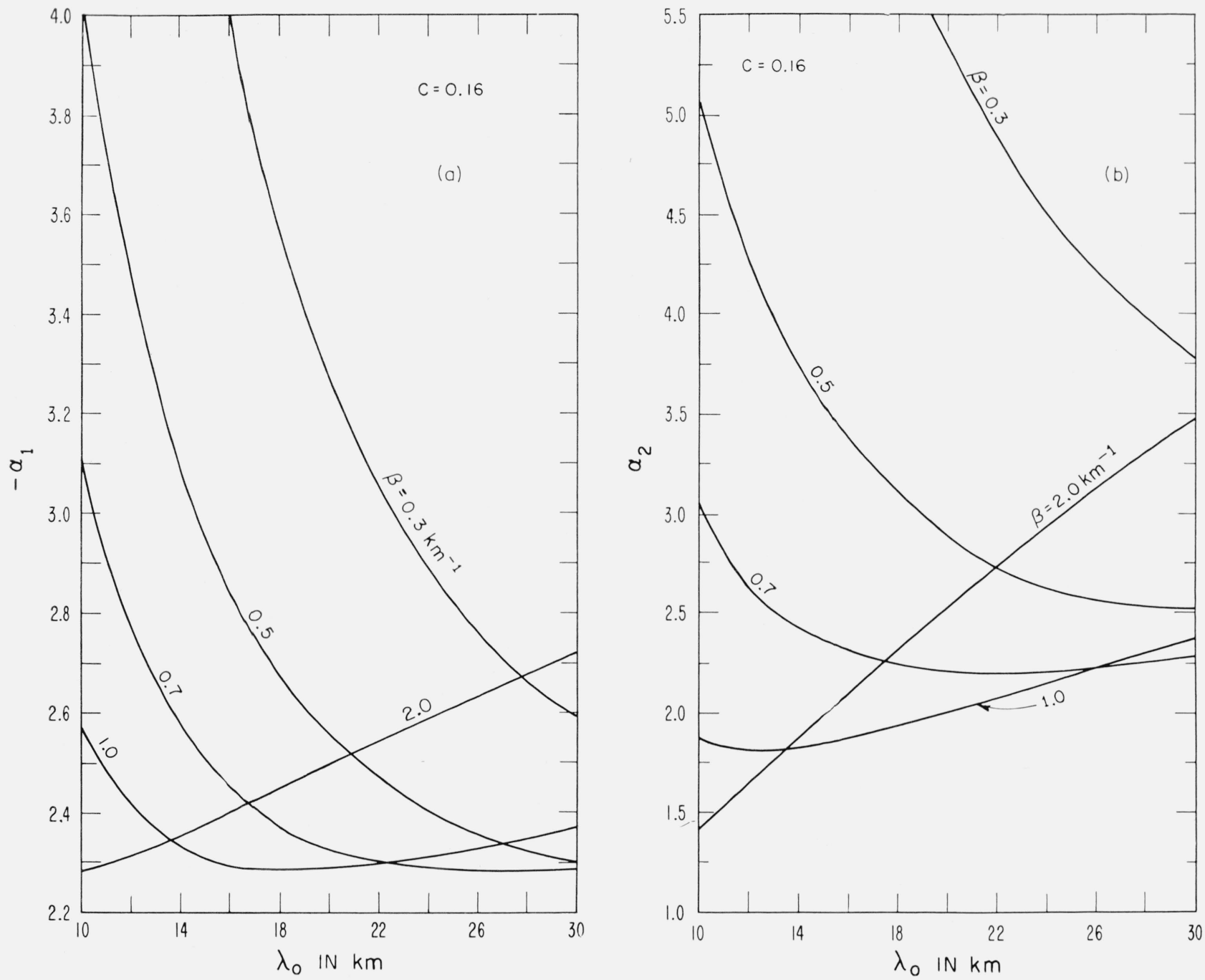

Figure 7. The real and imaginary parts of the function a plotted as a function of the wavelength $\lambda_{\mathrm{o}}$ for $\mathrm{C}=0.16$ and various values of $\beta$.

\section{References}

Barrington, R., B. Landmark, O. Holt, and E. Thrane (June 1962), Experimental studies of the ionospheric D-region, Report No. 44, Norwegian Defence Research Establishment, Kjeller, Norway.

Budden, K. G. (1961), Radio waves in the ionosphere (Cambridge University Press).

Crombie, D. D. (Sept.-Oct.1961), Reflection from a sharply bounded ionsophere for VLF propagation perpendicular to the magnetic meridian, J. Res. NBS 65D (Radio Prop.) $45.5-463$.

Johler, J. R., and J. D. Harper, Jr. (Jan.-Feb. 1962). Reflection and transmission of radio waves at a continuously stratified plasma with arbitrary magnetic induction, J. Res. NBS 66D (Radio Prop.) 81-99.
Kane, J. A. (1962). Re-evaluation of ionospheric electron densities and collision frequencies derived from rocket measurements, Chapter 29 in Radio Wave Absorption in the Ionosphere (Pergamon Press, Oxford).

Wait, J. R. (1962). Electromagnetic Waves in Stratified Media (Pergamon Press Ltd., Oxford). (Extensive references to related work are given.) 